\title{
Serine/Threonine-Protein Kinase ULK1
}

National Cancer Institute

\section{Source}

National Cancer Institute. Serine/Threonine-Protein Kinase ULK1. NCI Thesaurus. Code C116614.

Serine/threonine-protein kinase ULK1 (1050 aa, 113 kDa) is encoded by the human ULK1 gene. This protein is involved in protein phosphorylation that mediates the autophagocytotic process. 